Research Paper

\title{
Incidence and Risk Factors of Acute Kidney Injury after Radical Cystectomy: Importance of Preoperative Serum Uric Acid Level
}

Kyoung-Woon Joung, Seong-Soo Choi, Yu-Gyeong Kong, Jihion Yu, Jinwook Lim, Jai-Hyun Hwang, Young-Kug Kim ${ }^{\bowtie}$

Department of Anesthesiology and Pain Medicine, Asan Medical Center, University of Ulsan College of Medicine, Seoul, Korea

$\triangle$ Corresponding author: Young-Kug Kim, M.D., Ph.D., Professor, Department of Anesthesiology and Pain Medicine, Asan Medical Center, University of Ulsan College of Medicine, 88 Olympic-ro 43-gil, Songpa-gu, Seoul, 138-736, Republic of Korea. Tel.: +82-2-3010-5976; Fax: +82-2-3010-6790; E-mail: kyk@amc.seoul.kr

(C) 2015 Ivyspring International Publisher. Reproduction is permitted for personal, noncommercial use, provided that the article is in whole, unmodified, and properly cited. See http://ivyspring.com/terms for terms and conditions.

Received: 2015.03.11; Accepted: 2015.07.06; Published: 2015.07.16

\begin{abstract}
Background: Acute kidney injury (AKI) is a common complication after surgery and increases costs, morbidity, and mortality of hospitalized patients. While radical cystectomy associates significantly with an increased risk of serious complications, including AKI, risk factors of AKI after radical cystectomy has not been reported. This study was performed to determine the incidence and independent predictors of AKI after radical cystectomy.

Methods: All consecutive patients who underwent radical cystectomy in 2001-2013 in a single tertiary-care center were identified. Their demographics, laboratory values, and intraoperative data were recorded. Postoperative AKI was defined and staged according to the Acute Kidney Injury Network criteria on the basis of postoperative changes in creatinine levels. Independent predictors of AKI were identified by univariate and multivariate logistic regression analyses.

Results: Of the 238 patients who met the eligibility criteria, 91 (38.2\%) developed AKI. Univariate logistic regression analyses showed that male gender, high serum uric acid level, and long operation time associated with the development of AKI. On multivariate logistic regression analysis, preoperative serum uric acid concentration (odds ratio $[\mathrm{OR}]=1.251 ; 95 \%$ confidence interval $[\mathrm{Cl}]=$ $1.048-1.493 ; \mathrm{P}=0.013)$ and operation time $(\mathrm{OR}=1.005 ; 95 \% \mathrm{Cl}=1.002-1.008 ; \mathrm{P}=0.003)$ remained as independent predictors of AKI after radical cystectomy.

Conclusions: AKI after radical cystectomy was a relatively common complication. Its independent risk factors were high preoperative serum uric acid concentration and long operation time. These observations can help to prevent AKI after radical cystectomy.
\end{abstract}

Key words: acute kidney injury, radical cystectomy, uric acid

\section{Introduction}

Radical cystectomy is a definitive treatment for high-grade muscle-invasive bladder cancer. However, it associates with significant serious medical (e.g., renal insufficiency, cardiovascular complications, pulmonary complications, and sepsis), surgical (e.g., uretero-intestinal anastomotic stricture and reservoir rupture/perforation), metabolic (e.g., metabolic acidosis), and functional (e.g., urinary incontinence and chronic retention) complications.[1, 2] These postoperative complications could be reduced by improving the perioperative management of the patient, thereby promoting good patient outcomes after radical cystectomy.

One of the complications after surgery, including radical cystectomy, is acute kidney injury (AKI). AKI is characterized by an abrupt and sustained reduction 
in renal function, and increases the costs, morbidity, and mortality of hospitalized patients. [3, 4] When defined according to Acute Kidney Injury Network (AKIN) criteria (mainly increased serum creatinine levels and decreased urine output), the incidence of AKI after cardiac surgery is $27.9 \%$ and the 5 year mortality rate is $26.5 \%$.[5] Since the definitive treatment for postoperative AKI has not been established, it is essential to prevent it or detect it early. To improve the preventive management for AKI, a better understanding of the risk factors for postoperative AKI is needed.

Little is known about the risk factors that associate with AKI after radical cystectomy. Therefore, the present study was performed to evaluate the incidence and independent risk factors of AKI after radical cystectomy. For this purpose, postoperative AKI was defined by using AKIN criteria.

\section{Materials and Methods}

\section{Study population}

A retrospective review of the computerized patient record system of our hospital was performed to identify all consecutive patients who underwent radical cystectomy at our tertiary-care institution in Seoul, Korea between January 1, 2001 and December 31,2013 . Patients who met the following criteria were excluded: age below 20 years and incomplete preoperative and postoperative laboratory data missing either one of following, C-reactive protein, estimated glomerular filtration rate (eGFR), uric acid, and serum creatinine. In addition, we excluded patients with preoperative end stage renal disease. The demographic, clinical, and intraoperative and postoperative data were collected from the computerized databases. The study protocol was approved by our institutional review board.

\section{Anesthetic and surgical technique}

Anesthetic techniques were performed according to institutional standards. General anesthesia was induced by using a bolus intravenous (IV) injection of pentotal sodium $(5 \mathrm{mg} / \mathrm{kg}$ ) or propofol $(2 \mathrm{mg} / \mathrm{kg})$. In all but 7 patients, the general anesthesia was maintained with volatile anesthetics (isoflurane, sevoflurane, or desflurane). The remaining 7 patients were maintained with a continuous infusion of propofol and remifentanil that was delivered by a target control infusion pump (Orchestra ${ }^{\circledR}$ Base Prima; Fresenius Kabi, Brezins, France). To facilitate orotracheal intubation, all patients received a bolus IV injection of $0.5-0.8 \mathrm{mg} / \mathrm{kg}$ of rocuronium.

Crystalloid (lactated Ringer's solution or plasmalyte) and colloid solution (Voluven ${ }^{\circledR}, 6 \%$ hydroxy- ethyl starch 130/0.4) were administered during surgery. Arterial blood pressure during anesthesia was maintained at above $65 \mathrm{mmHg}$ of mean arterial pressure or above $90 \mathrm{mmHg}$ of systolic arterial pressure. Furosemide was administered intravenously if the central venous pressure exceeded $10 \mathrm{mmHg}$. Packed red blood cell transfusion was performed during the perioperative period if the hemoglobin concentration reached $<8 \mathrm{~g} / \mathrm{dL}$.

As described previously,[6] all surgical procedures were performed by experienced surgeons. In all cases, the surgeon and the patient chose together which type of urinary diversion would be used. All patients underwent orthotopic or non-orthotopic urinary diversion, except the patient who have the presence of absolute contraindications of urinary diversion.

\section{Measurements and definitions}

The data that were collected included the demographic data, laboratory values, intraoperative data, and postoperative outcomes. Anemia was defined by serum hemoglobin concentration $<13.0 \mathrm{~g} / \mathrm{dL}$ in man and $<12.0 \mathrm{~g} / \mathrm{dL}$ in female. Hypertension was defined as systolic blood pressure $>140 \mathrm{mmHg}$, diastolic blood pressure $>90 \mathrm{mmHg}$, or medication with an anti-hypertensive drug. Heart failure was defined as a history of any type of heart failure that was diagnosed by a cardiologist with/without medication or decreased ejection fraction $(<40 \%)$. Cerebrovascular disease was defined as a history of carotid artery stent or angioplasty, transient ischemic attack, stroke, or cerebral hemorrhagic event. Central venous pressure was evaluated as an average of three values (after induction of general anesthesia, at the end of bladder excision, and at the end of surgery). Preoperative renal function was defined as the eGFR, which was calculated using the Modification of Diet in Renal Disease equation II $(\mathrm{eGFR}=186 \times$ serum creatinine $^{-1.154} \times$ age $^{-0.203} \times[0.742$ if female $] \times[1.210$ if African-American]).[7]

Postoperative AKI after radical cystectomy was defined and staged according to the AKIN criteria on the basis of postoperative change in serum creatinine levels. Stage I was defined as an increase to $\geq 0.3$ $\mathrm{mg} / \mathrm{dL}$ or $150-200 \%$ of baseline values. Stage II was defined as an increase of $201-300 \%$ relative to baseline values. Stage III was defined as an increase of $>300 \%$ relative to baseline values, an increase to $\geq 4.0 \mathrm{mg} / \mathrm{dL}$ with an acute increase of at least $0.5 \mathrm{mg} / \mathrm{dL}$, or the need for renal replacement therapy.[8] The urine output criteria of AKIN were not used in the present study. Patients who underwent radical cystectomy with neobladder reconstruction were received massive and frequent bladder irrigation (normal saline 
3-4 L/day), at least, until postoperative 2 weeks. And, hourly urine output was not checked for patients transferred to general ward postoperatively. Moreover, intraoperative use of diuretics also can make it inconsistent to detect AKI by urine output criteria.[9] Thus, the application of urine output criteria is incoherent and inaccurate to evaluate postoperative AKI in these patients.

Postoperative outcomes included length of hospital stay and the Clavien-Dindo classification system.[10]

\section{Statistical analysis}

All continuous variables were expressed as mean \pm standard deviation or median with interquartile range. Categorical variables were expressed as number and percentage. To compare the patients with and without AKI in terms of demographics and intraoperative characteristics, Student's t-test or the Mann-Whitney U test were used for continuous variables, while the Chi-square test or Fisher's exact test were used for categorical variables. To identify independent risk factors for AKI after radical cystectomy, logistic regression analysis was performed. All factors that had a $P$ value $<0.05$ on univariate logistic regression analysis were included in a stepwise multivariate logistic regression analysis. To summarize the strength of the association of each variable with postoperative AKI, odds ratios (ORs) with 95\% confidence interval $(\mathrm{CI})$ were calculated. To evaluate model calibration, the Hosmer-Lemeshow statistic was used. All reported $\mathrm{P}$ values were two-sided. $\mathrm{P}$ values $<0.05$ were considered to indicate statistical significance. All data manipulations and statistical analyses were performed by using SPSS ${ }^{\circledR}$ Version 21.0 software.

\section{Results}

The medical chart review identified 698 patients who underwent radical cystectomy during the study period. Of these, 460 were excluded because their age was below 20 years $(n=20)$ and they did not performed some laboratory examination such as C-reactive protein, eGFR, uric acid, and serum creatinine $(\mathrm{n}=399)$. Patients with end stage renal disease also excluded $(\mathrm{n}=41)$. The baseline and intraoperative characteristics of the remaining 238 patients are summarized in Tables 1 and 2, respectively. Of these, $91(38.2 \%)$ developed postoperative AKI. They were more likely to have higher baseline serum uric acid levels than the patients who did not develop postoperative AKI (Table 1). Their surgical times were longer than the patients without AKI (Table 2).

In addition, there were no significant differences in hematological and biochemical variables such as hematocrit, platelet count, albumin, aspartate aminotransferase, alanine transaminase, sodium, potassium, and chloride levels between no-AKI and AKI groups until postoperative day 2 (data not shown). There was no significant difference in median length of hospital stay between two groups $(\mathrm{P}=0.152)$. Postoperative complications according to the Clavien-Dindo classifications were not significantly different between two groups $(\mathrm{P}=0.424)$.

Table 1. Baseline demographic and clinical characteristics of 238 patients who underwent radical cystectomy

\begin{tabular}{|c|c|c|c|c|}
\hline & $\begin{array}{l}\text { All patients } \\
(\mathrm{N}=238)\end{array}$ & $\begin{array}{l}\text { No-AKI group } \\
(\mathrm{n}=147)\end{array}$ & $\begin{array}{l}\text { AKI group } \\
(\mathrm{n}=91)\end{array}$ & P value \\
\hline Age (yr) & $63.8 \pm 9.6$ & $63.7 \pm 9.4$ & $63.9 \pm 10.0$ & 0.902 \\
\hline Male & $201(84.5 \%)$ & $118(80.3 \%)$ & $83(91.2 \%)$ & 0.017 \\
\hline Body mass index $\left(\mathrm{kg} / \mathrm{m}^{2}\right)$ & $24.2 \pm 3.15$ & $23.91 \pm 2.80$ & $24.41 \pm 3.14$ & 0.205 \\
\hline ASA & & & & 0.467 \\
\hline 1 and 2 & $185(77.7 \%)$ & $115(78.2 \%)$ & $70(76.9 \%)$ & \\
\hline 3 and 4 & $53(22.3 \%)$ & $32(21.8 \%)$ & $21(23.1 \%)$ & \\
\hline Anemia & $121(50.8 \%)$ & $75(51 \%)$ & $46(50.5 \%)$ & 0.525 \\
\hline Diabetes mellitus & $46(19.3 \%)$ & $31(21.1 \%)$ & $15(16.5 \%)$ & 0.242 \\
\hline Smoking & $104(43.7 \%)$ & $61(41.5 \%)$ & $43(47.3 \%)$ & 0.231 \\
\hline COPD & $6(2.5 \%)$ & $3(2.0 \%)$ & $3(3.3 \%)$ & 0.419 \\
\hline Hypertension & $103(43.3 \%)$ & $58(39.5 \%)$ & $45(49.5 \%)$ & 0.084 \\
\hline Heart failure & $2(0.8 \%)$ & $1(0.7 \%)$ & $1(1.1 \%)$ & 0.620 \\
\hline Cerebrovascular disease & $5(2.1 \%)$ & $3(2.0 \%)$ & $2(2.2 \%)$ & 0.634 \\
\hline Coronary artery disease & $10(4.2 \%)$ & $6(4.1 \%)$ & $4(4.1 \%)$ & 0.575 \\
\hline Hematocrit (\%) & 38.1 [33.3-41.8] & $37.8[32.5-41.8]$ & $38.3[33.8-41.8]$ & 0.478 \\
\hline Creatinine $(\mathrm{mg} / \mathrm{dL})$ & $1.13 \pm 0.88$ & $1.16 \pm 1.06$ & $1.08 \pm 0.49$ & 0.480 \\
\hline Bilirubin, total (mg/dL) & $0.65 \pm 0.32$ & $0.65 \pm 0.35$ & $0.65 \pm 0.26$ & 0.940 \\
\hline Albumin (g/dL) & $3.82 \pm 0.54$ & $3.84 \pm 0.56$ & $3.77 \pm 0.50$ & 0.336 \\
\hline Uric acid $(\mathrm{mg} / \mathrm{dL})$ & $5.33 \pm 1.60$ & $5.08 \pm 1.58$ & $5.73 \pm 1.59$ & 0.003 \\
\hline $\mathrm{CRP}(\mathrm{mg} / \mathrm{dL})$ & $0.28[0.10-0.73]$ & $0.27[0.10-0.51]$ & $0.34[0.10-0.92]$ & 0.017 \\
\hline eGFR $\left(\mathrm{mL} / \mathrm{min} / 1.73 \mathrm{~m}^{2}\right)$ & $57.7 \pm 10.8$ & $56.9 \pm 11.0$ & $58.9 \pm 10.5$ & 0.153 \\
\hline
\end{tabular}

The data are expressed as mean \pm standard deviation, number of patients (\%), or median [first-third quartiles]. AKI = acute kidney injury; ASA = American Society of Anesthesiologist Classification; $\mathrm{COPD}=$ chronic obstructive pulmonary disease; $\mathrm{CRP}=\mathrm{C}$-reactive protein; $\mathrm{eGFR}=$ estimated glomerular filtration rate. 
Table 2. Intraoperative data of 238 patients who underwent radical cystectomy

\begin{tabular}{llll}
\hline & $\begin{array}{l}\text { All patients } \\
(\mathrm{N}=238)\end{array}$ & $\begin{array}{l}\text { No-AKI group } \\
(\mathrm{n}=147)\end{array}$ & \multicolumn{1}{c}{$\begin{array}{l}\text { AKI group } \\
(\mathrm{n}=91)\end{array}$} \\
\hline Operation time (min) & $451.3[387.0-501.5]$ & $436.9[378.0-477.0]$ & $474.4[414.0-548.0]$ \\
Crystalloid (mL) & $3923.4 \pm 1431.6$ & $3900.0 \pm 1466.0$ & $3961.2 \pm 1381.4$ \\
Colloid (mL) & $810.7 \pm 393.6$ & $800.3 \pm 367.6$ & $827.5 \pm 434.0$ \\
CVP (mmHg) & $7.2 \pm 2.4$ & $7.3 \pm 2.4$ & $7.0 \pm 2.4$ \\
RBC transfused (U) & $2.6 \pm 2.3$ & $2.7 \pm 2.3$ & $2.3 \pm 2.2$ \\
Use of FFP & $18(7.6 \%)$ & $11(7.5 \%)$ & $7(7.7 \%)$ \\
Use of platelet concentrates & $3(1.3 \%)$ & $2(1.4 \%)$ & 0.749 \\
Use of diuretics & $12(5.0 \%)$ & $7(4.8 \%)$ & 0.605 \\
\hline
\end{tabular}

The data are expressed as mean \pm standard deviation, number of patients (\%), or median [first-third quartiles]. *Central venous pressure was evaluated as the average of three values (after induction of general anesthesia, at the end of bladder excision, and at the end of surgery). AKI = acute kidney injury; CVP = central venous pressure; RBC $=$ red blood cell; FFP $=$ fresh frozen plasma.

Table 3. Univariate and multivariate regression analyses to identify factors that associate with acute kidney injury after radical cystectomy

\begin{tabular}{|c|c|c|c|c|}
\hline & \multicolumn{2}{|c|}{ Univariate analysis } & \multicolumn{2}{|c|}{ Multivariate analysis } \\
\hline & Odds ratio $(95 \% \mathrm{CI})$ & P value & Odds ratio $(95 \% \mathrm{CI})$ & P value \\
\hline Sex (female/male) & $0.392(0.171-0.901)$ & 0.027 & & \\
\hline Hypertension & $1.501(0.886-2.544)$ & 0.131 & & \\
\hline Serum uric acid & $1.297(1.089-1.546)$ & 0.004 & $1.251(1.048-1.493)$ & 0.013 \\
\hline CRP & $0.851(0.722-1.002)$ & 0.053 & & \\
\hline eGFR & $1.019(0.992-1.047)$ & 0.159 & & \\
\hline Operation time & $1.004(1.001-1.007)$ & 0.003 & 1.005 (1.002-1.008) & 0.003 \\
\hline RBC transfused & $0.908(0.805-1.025)$ & 0.118 & & \\
\hline
\end{tabular}

$\mathrm{CI}=$ confidence interval; $\mathrm{CRP}=\mathrm{C}$-reactive protein; $\mathrm{eGFR}=$ estimated glomerular filtration rate; $\mathrm{RBC}=$ red blood cell.

Univariate logistic regression analysis revealed that male gender, high baseline serum uric acid level, and a long operation time associated with the development of AKI (Table 3). Multivariate logistic regression analysis revealed that independent risk factors for postoperative AKI were high baseline serum uric acid concentration $(\mathrm{OR}=1.251 ; 95 \%$ CI 1.048-1.493; $\mathrm{P}$ $=0.013)$ and a long operation time $(\mathrm{OR}=1.005 ; 95 \% \mathrm{CI}$ $=1.002-1.008 ; \mathrm{P}=0.003)$ (Table 3$)$.

\section{Discussion}

The present study showed that $38.2 \%$ of the patients who underwent radical cystectomy developed postoperative AKI and that this complication associated independently with high baseline serum uric acid concentration and long operation time.

Bladder cancer is the ninth most common cancer. It develops more frequently in men than in women,[11] and its most common histological type is transitional cell carcinoma. Approximately $30 \%$ of bladder cancers have invaded the muscle at the time of diagnosis.[12, 13] While non-muscle-invasive bladder cancer is not life threatening, muscle-invasive bladder cancer associates with a high risk of distant metastasis and death. The standard treatment of muscle-invasive bladder cancer is radical cystectomy and urinary diversion. The complication rate after radical cystectomy ranges between $20 \%$ and 58\%.[14-17] These complications include ileus, wound dehiscence, urinary tract infection, and renal insufficiency.[18]
With regard to the latter complication, a previous study showed that between $4 \%$ and $7 \%$ of patients who undergo radical cystectomy develop acute renal failure (ARF).[19] Similarly, a recent observational study reported that $6.7 \%$ of patients who underwent urological surgery developed postoperative AKI.[20] By contrast, the present study found an incidence of AKI after radical cystectomy of $38.2 \%$. This disparity may relate to the criteria that were used to detect the kidney injury and the nature of the patient population. ARF is defined as severe kidney injury that requires renal replacement therapy. On the other hand, AKI is defined on the basis of relatively small changes in creatinine levels relative to baseline. Thus, the use of AKI criteria would lead to a considerably higher incidence of kidney injury than if the classical ARF criteria were used.[21] The relatively high incidence of AKI that was detected by the present study is important because it is well-known that postoperative AKI increases costs, morbidity, and mortality.[3, 4] Thus, physicians should be aware of the strong possibility that AKI may develop after radical cystectomy.

Since definitive treatments for postoperative AKI have not been established, it is important to identify risk factors that will facilitate the early detection of AKI and aid risk management strategies. The present study showed that a higher preoperative serum uric acid level was an independent risk factor for AKI after radical cystectomy. It was shown previously that patients with a large tumor burden and those receiving 
chemotherapy can develop uric acid-induced AKI. In such patients, AKI is caused by hyperuricemia and the intratubular deposition of uric acid crystals after the rapid release of nucleotides after tumor cell death.[22, 23] However, several recent studies showed that preoperative hyperuricemia may also associate with an increased risk of postoperative AKI even if intratubular uric acid crystal deposition does not occur. One of these studies was by Ejaz et al.,[24] who reported that elevated preoperative serum uric acid may be a risk factor for AKI in patients undergoing high-risk cardiovascular surgery. The other study was an observational study by our center that also suggested that preoperative hyperuricemia is an independent risk factor for AKI after cardiovascular surgery.[25] The possible mechanisms by which uric acid induces AKI are renal vasoconstriction, endothelial dysfunction, impairment of renal auto-regulation, and tubular obstruction by uric acid crystals. $[23,26]$ With regard to endothelial dysfunction, uric acid activates intracellular protein kinases (p38 and extracellular-signal-regulated kinases 1/2) and nuclear transcription factors (nuclear factor- $\mathrm{kB}$ and activator protein-1), thereby stimulating vascular smooth muscle cell proliferation and local inflammation.[27, 28] Hyperuricemia also seems to stimulate the production of proinflammatory substances, such as C-reactive protein, interleukin-1, interleukin-6, and tumor necrosis factor $\mathrm{a}-2$, which may further promote endothelial dysfunction. Uric acid also appears to activate the renin-angiotensin system by up-regulating the expression of angiotensinogen, angiotensin-converting enzyme, and angiotensin II receptor expression. This suppresses nitric oxide synthesis and increases vascular tone.[29, 30]

The present study also showed that a longer operation time associated independently with the development of AKI after radical cystectomy. Similarly, Tomas et al.[31] reported that a longer operation time associated independently with a higher overall incidence of complications after radical cystectomy. Prolonged operation time also associates with a greater risk of AKI after surgical procedures for lung cancer.[32] A long surgical time may reflect the need for complex surgical procedures that may directly and/or indirectly damage the kidney.

The present observational study has some possible limitations. First, it was a retrospective observational study. Although we considered many variables and performed multivariate analysis to obtain reliable results, we cannot exclude the possibility that factors that were not evaluated may have influenced the outcomes. Second, large numbers of patients were excluded in the present study. Although it might act as a selection bias, the quality of data and reliability of analysis could be increased. Third, we measured the serum uric acid levels preoperatively and only once. Although these levels associated significantly with AKI in this study, we cannot exclude the possibility that uric acid levels at other time points may also associate with the risk of AKI. Indeed, Ejaz et al.[33] reported that postoperative serum uric acid levels associate with a higher risk of AKI after cardiac surgery. Further studies investigating the association of uric acid levels with AKI risk after radical cystectomy should include serial measurements of serum uric acid in the preoperative and postoperative periods.

In conclusion, the present study showed that AKI was a common complication after radical cystectomy and that it associated with a higher preoperative serum uric acid level and a longer operation time. The identification of these risk factors can be useful for preventing the development of AKI after radical cystectomy.

\section{Competing Interests}

The authors have declared that no competing interest exists.

\section{References}

[1] Ali-el-Dein B, Shaaban AA, Abu-Eideh RH, et al. Surgical complications following radical cystectomy and orthotopic neobladders in women. J Urol. 2008;180: 206-10.

[2] Chahal R, Sundaram SK, Iddenden R, et al. A study of the morbidity, mortality and long-term survival following radical cystectomy and radical radiotherapy in the treatment of invasive bladder cancer in Yorkshire. Eur Urol. 2003;43: 246-57.

[3] Chertow GM, Burdick E, Honour M, et al. Acute kidney injury, mortality, length of stay, and costs in hospitalized patients. J Am Soc Nephrol. 2005;16: 3365-70.

[4] Hobson C, Ozrazgat-Baslanti T, Kuxhausen A, et al. Cost and Mortality Associated With Postoperative Acute Kidney Injury. Ann Surg. 2015;261: 1207-14.

[5] Hansen MK, Gammelager H, Mikkelsen MM, et al. Post-operative acute kidney injury and five-year risk of death, myocardial infarction, and stroke among elective cardiac surgical patients: a cohort study. Critical Care. 2013;17: R292.

[6] Jeong IG, You D, Kim J, et al. Factors associated with non-orthotopic urinary diversion after radical cystectomy. World J Urol. 2012;30: 815-20.

[7] Levey AS, Bosch JP, Lewis JB, et al. A more accurate method to estimate glomerular filtration rate from serum creatinine: a new prediction equation. Modification of Diet in Renal Disease Study Group. Ann Intern Med. 1999;130: 461-70.

[8] Mehta RL, Kellum JA, Shah SV, et al. Acute Kidney Injury Network: report of an initiative to improve outcomes in acute kidney injury. Crit Care. 2007;11: R31.

[9] Elhmidi Y, Bleiziffer S, Piazza N, et al. Incidence and predictors of acute kidney injury in patients undergoing transcatheter aortic valve implantation. Am Heart J. 2011;161: 735-9.

[10] Dindo D, Demartines N, Clavien PA. Classification of surgical complications: a new proposal with evaluation in a cohort of 6336 patients and results of a survey. Ann Surg. 2004;240: 205-13.

[11] Ferlay J, Shin HR, Bray F, et al. Estimates of worldwide burden of cancer in 2008: GLOBOCAN 2008. Int J Cancer. 2010;127: 2893-917.

[12] Di Stasi SM, Giannantoni A, Stephen RL, et al. Intravesical electromotive mitomycin $C$ versus passive transport mitomycin $C$ for high risk superficial bladder cancer: a prospective randomized study. J Urol. 2003;170: 777-82.

[13] Kaufman DS, Shipley WU, Feldman AS. Bladder cancer. Lancet. 2009;374: 239-49.

[14] Killeen KP, Libertino JA. Management of bowel and urinary tract complications after urinary diversion. Urol Clin North Am. 1988;15: 183-94.

[15] Lawrentschuk N, Colombo R, Hakenberg OW, et al. Prevention and management of complications following radical cystectomy for bladder cancer. Eur Urol. 2010;57: 983-1001.

[16] Madersbacher S, Mohrle K, Burkhard F, et al. Long-term voiding pattern of patients with ileal orthotopic bladder substitutes. J Urol. 2002;167: 2052-7. 
[17] Perimenis P, Burkhard FC, Kessler TM, et al. Ileal orthotopic bladder substitute combined with an afferent tubular segment: long-term upper urinary tract changes and voiding pattern. Eur Urol. 2004;46: 604-9.

[18] Kim SH, Yu A, Jung JH, et al. Incidence and risk factors of 30-day early and 90-day late morbidity and mortality of radical cystectomy during a 13-year follow-up: a comparative propensity-score matched analysis of complications between neobladder and ileal conduit. Jpn J Clin Oncol. 2014;44: 677-85.

[19] Froehner M, Brausi MA, Herr HW, et al. Complications following radical cystectomy for bladder cancer in the elderly. Eur Urol. 2009;56: 443-54.

[20] Caddeo G, Williams ST, McIntyre CW, et al. Acute kidney injury in urology patients: incidence, causes and outcomes. Nephrourol Mon. 2013;5: 955-61.

[21] Bove T, Monaco F, Covello RD, et al. Acute renal failure and cardiac surgery. HSR Proc Intensive Care Cardiovasc Anesth. 2009;1: 13-21.

[22] Cohen LF, Balow JE, Magrath IT, et al. Acute tumor lysis syndrome. A review of 37 patients with Burkitt's lymphoma. Am J Med. 1980;68: 486-91.

[23] Shimada M, Johnson RJ, May WS, Jr., et al. A novel role for uric acid in acute kidney injury associated with tumour lysis syndrome. Nephrol Dial Transplant. 2009;24: 2960-4.

[24] Ejaz AA, Beaver TM, Shimada M, et al. Uric acid: a novel risk factor for acute kidney injury in high-risk cardiac surgery patients? Am J Nephrol. 2009;30: 425-9.

[25] Joung KW, Jo JY, Kim WJ, et al. Association of preoperative uric acid and acute kidney injury following cardiovascular surgery. J Cardiothorac Vasc Anesth. 2014;28: 1440-7.

[26] Kim YG, Huang XR, Suga $S$, et al. Involvement of macrophage migration inhibitory factor (MIF) in experimental uric acid nephropathy. Mol Med. 2000;6: 837-48

[27] Filiopoulos V, Hadjiyannakos D, Vlassopoulos D. New Insights into Uric Acid Effects on the Progression and Prognosis of Chronic Kidney Disease. Renal Failure. 2012;34: 510-20.

[28] Sautin YY, Nakagawa T, Zharikov $S$, et al. Adverse effects of the classic antioxidant uric acid in adipocytes: NADPH oxidase-mediated oxidative/nitrosative stress. Am J Physiol Cell Physiol. 2007;293: C584-96.

[29] Yu MA, Sanchez-Lozada LG, Johnson RJ, et al. Oxidative stress with an activation of the renin-angiotensin system in human vascular endothelial cells as a novel mechanism of uric acid-induced endothelial dysfunction. J Hypertens. 2010;28: 1234-42.

[30] Ingelfinger JR, Dzau VJ. Molecular-Biology of Renal Injury: Emphasis on the Role of the Renin-Angiotensin System. J Am Soc Nephrol. 1991;2: S9-S20.

[31] Jerlstrom T, Gardmark T, Carringer M, et al. Urinary bladder cancer treated with radical cystectomy: Perioperative parameters and early complications prospectively registered in a national population-based database. Scand J Urol. 2014;48: 334-40

[32] Licker M, Cartier V, Robert J, et al. Risk factors of acute kidney injury according to RIFLE criteria after lung cancer surgery. Ann Thorac Surg. 2011;91: 844-50.

[33] Ejaz AA, Kambhampati G, Ejaz NI, et al. Post-operative serum uric acid and acute kidney injury. J Nephrol. 2012;25: 497-505. 